\title{
A prospective study of critical methods of amniotic fluid volume assessment
}

\author{
Mayur Rajendra Gandhi*
}

Department of Obstetrics and Gynecology, GMERS Medical College, Dharpur-Patan, Gujarat, India

Received: 29 April 2017

Accepted: 22 May 2017

\section{*Correspondence:}

Dr. Mayur Rajendra Gandhi,

E-mail: maydeep2008@yahoo.com

Copyright: () the author(s), publisher and licensee Medip Academy. This is an open-access article distributed under the terms of the Creative Commons Attribution Non-Commercial License, which permits unrestricted non-commercial use, distribution, and reproduction in any medium, provided the original work is properly cited.

\section{ABSTRACT}

Background: Adequate amniotic fluid volume (AFV) is required for fetal well-being and its assessment is taken as the single most important variable in fetal biophysical scoring system. I have critically evaluated various methods of amniotic fluid volume assessment keeping Amniotic Fluid Index (AFI) as the standard technique of Amniotic Fluid Volume (AFV) assessment.

Methods: This was a prospective study where all patients were assessed for amniotic fluid volume at one sitting first by abdominal palpation followed by ultrasonographic assessment by various methods. Critical analysis of each individual method of amniotic fluid volume assessment was carried out for its sensitivity and specificity with regard to oligohydramnios, polyhydramnios and euamnios keeping AFI as the standard technique of AFV assessment. Effort was made to find out the best possible method of AFV assessment.

Results: Almost all methods are reasonably effective in assessment of normal AFV. For assessment of oligohydraamnios $2 \mathrm{~cm} \times 2 \mathrm{~cm}$ pocket and Maximum Vertical Pocket (MVP) depth (of $2 \mathrm{~cm}$ rule) methods are most suitable whereas other methods were found to be inappropriate. For assessment of polyhydramnios with regard to specificity MVP depth $(>8 \mathrm{~cm})$ is reliable but with regard to sensitivity, subjective assessment and abdominal palpation are more reliable.

Conclusions: When we compare all methods of AFV assessment, AFI and MVP depth (of $2 \mathrm{~cm}$ rule) have better correlation. Hence these methods are suggested for AFV studies in current clinical settings.

Keywords: AFV, AFI, MVP depth

\section{INTRODUCTION}

Amniotic fluid volume is an important parameter to assess fetal well-being, growth and development. Appreciation of the importance of amniotic fluid volume as an indicator of fetal status is a relatively recent development. ${ }^{1}$ The amniotic fluid is fundamental for proper fetal development and growth, and amniotic fluid volume using prenatal ultrasound have become standard in fetal surveillance, especially in the evaluation of high risk pregnancies. In the course of pregnancy, the subject of amniotic fluid is seldom considered unless there is some abnormality e.g. polyhydramnios, oligohydramnios or the occurrence of meconium staining. Sonographic evaluation of the amniotic fluid can thus aid in the diagnosis of fetal structural anomalies and fetal compromise, and can help in making pregnancy management decisions. ${ }^{2}$

However, when amniotic fluid volume is abnormal, perinatal morbidity and mortality are greatly increased. The perinatal mortality was 2-fold more in pregnancies 
with polyhydramnios as compared to pregnancies with normal amniotic fluid volume. The perinatal mortality was 13-fold more than normal when amniotic fluid was sonographically marginal (borderline). ${ }^{3}$ From above figures, one can understand that adequate amniotic fluid volume is required for fetal well-being and its assessment is taken as the most important variable in fetal biophysical scoring system. Despite a significant number of published studies that address each of these concerns, the single best answer to guide us in the use of this assessment for clinical management of patients has not been determined. I have tried to find out the best possible method of amniotic fluid volume assessment in this study.

\section{METHODS}

This was a prospective study carried out on 151 registered patients in the Department of Obstetrics and Gynecology, Civil Hospital, Ahmedabad during one and half year period from January 97 to June 98. Most of the patients were admitted in our antenatal ward due to one or other reasons; however, few outdoor patients were also registered for this study. All were singleton pregnancy and the selection of cases was random. All were term patients i.e. 37 completed weeks according to last menstrual period and up to 42 weeks. All patients were assessed at one sitting, first by abdominal palpation followed immediately by sonographic assessment. They were all delivered at Civil Hospital, Ahmedabad. Assessment of AFV was done by various methods. Assessment of amniotic fluid volume by abdominal palpation was done and patients were grouped as having AFV oligohydramnios, borderline (less than normal), normal (adequate) and polyhydramnios (increased). In this method of assessment, the factors to be considered include size of the uterus, difficulty or ease with which fetal parts are felt through uterine wall and the mobility of fetal parts within the uterine cavity. In the past obstetrians relied on abdominal palpation and fundal height to detect abnormal intrauterine volumes. ${ }^{4}$

Assessment of amniotic fluid volume by ultrasound (subjective nonquantitative method) was done and patients were grouped as having AFV normal, reduced or absent. In this method, the fetus is scanned longitudinally in the area of the fetal limbs. Assessment of an echo free space between the limbs and the uterine wall anteriorly or posteriorly is made. Demonstration of such a space between the limbs and between the limbs and the uterine wall qualifies the volume as normal or adequate. Lack of space between the limbs and the walls of the uterus but an echo free space between adjacent limbs qualifies the volume as reduced or borderline-low.

Absent of echo free space in the area of the fetal limbs qualifies the volume as absent. Crowley $\mathrm{P}$ described a systematic evaluation of the amniotic fluid, which was then correlated with poor outcome in patients meeting criteria for absent amniotic fluid. ${ }^{5}$ Assessment of amniotic fluid volume by ultrasound (semi-quantitative method) was done using AFI $(\mathrm{cm})$ by four quadrant sum totals of largest vertical dimensions. ${ }^{6}$ Patients were grouped as oligohydramnios, borderline (less than normal), normal (adequate) and polyhydramnios (increased). Assessment of amniotic fluid volume by ultrasound (semiquantitative method) was done using measurement of the single largest pocket in two perpendicular planes $(2 \mathrm{~cm} \times 2 \mathrm{~cm}$ pocket method) which was first described by Manning et $\mathrm{al}^{7}$ as part of the fetal biophysical profile.

Normal amniotic fluid volume was defined as amniotic fluid visible throughout the uterine cavity with the largest pocket greater than $2 \mathrm{~cm}$ in two perpendicular planes. Oligohydramnios was defined as single pocket that measured $<2 \mathrm{~cm}$ in both the vertical and horizontal plane. Assessment of amniotic fluid volume by ultrasound (semiquantitative method) was done by MVP depth described by Chamberlin et $\mathrm{al}^{3}$ in which the single deepest uninterrupted pocket of amniotic fluid is measured. Assessment of amniotic fluid volume by ultrasound (semiquantitative method) was done by measurement of two diameter single pocket which is obtained by multiplication of vertical and horizontal diameters of the largest pocket. All patients were admitted to labour room, detailed history, general examination, local examination was carried out.

\section{RESULTS}

All data were tabulated and analysed by appropriate biostatical test using EPI INFO version 6.04 DP value of 0.05 was considered as significant. I have included observations of the present study and also its comparison with other similar studies. In this study, data derived from AFI was considered basic and data derived from other methods were compared with AFI data. Table 1 shows analysis of amniotic fluid volume by amniotic fluid index. It shows that in present study out of 151 patients, 17 patients were detected having oligohydramnios, 28 patients having borderline amniotic fluid volume, 95 patients having normal amount of amniotic fluid volume and 11 patients having polyhydramnios. ${ }^{8}$

Table 1: AVF by AFI related analysis.

\begin{tabular}{|lllll|}
\hline AFV & $\begin{array}{l}\text { AFI } \\
(\mathrm{cm})\end{array}$ & $\begin{array}{l}\text { Present } \\
\text { study } \\
\text { \% }\end{array}$ & $\begin{array}{l}\text { Phallen } \\
\text { et al \% }\end{array}$ \\
\hline Oligohydramnios & $\begin{array}{l}0-5 \mathrm{~cm} \\
17\end{array}$ & 11.26 & 08 \\
\hline Borderline & $\begin{array}{l}5.1- \\
8.0 \mathrm{~cm}\end{array}$ & 28 & 18.54 & 20 \\
\hline Normal & $\begin{array}{l}8.1- \\
18 \mathrm{~cm}\end{array}$ & 95 & 62.92 & 66 \\
\hline Polyhydramnios & $>18 \mathrm{~cm}$ & 11 & 07.28 & 06 \\
\hline
\end{tabular}

Table 2 shows analysis of amniotic fluid volume by AFI according to age groups. It shows maximum number of 
patients were from age group of 20-24 years and next in the age group of 25-29 years.

Table 3 shows that in present study out of 151 patients, while assessment of amniotic fluid volume by MVP depth 19 patients were detected having borderline amniotic fluid volume, 130 patients having normal amount of amniotic fluid volume and 02 patients having polyhydramnios were detected and number of patients with oligohydramnios was zero. ${ }^{1,3}$

Table 2: Age and AFI related analysis $(\mathrm{N}=151)$.

\begin{tabular}{|c|c|c|c|c|}
\hline Age & Amniotic fluid inde & & & \\
\hline & 0-5 CM N=17 (\%) & 5.1-8 CM N=28(\%) & 8.1-18 CM N=95 (\%) & $>18 \mathrm{CM} \mathrm{N}=11(\%)$ \\
\hline$<20$ Years & $02(11.76)$ & $01(03.57)$ & $01(01.05)$ & - \\
\hline 20-24 Years & $07(41.18)$ & $14(50.00)$ & $42(44.21)$ & $07(63.64)$ \\
\hline 25-29 Years & $03(17.65)$ & $12(42.86)$ & $35(36.84)$ & $01(09.09)$ \\
\hline 30-34 Years & $03(17.65)$ & - & $14(14.74)$ & $02(18.18)$ \\
\hline$>34$ Years & $02(17.65)$ & $01(03.57)$ & $03(03.16)$ & $01(09.09)$ \\
\hline
\end{tabular}

Table 3: AVF by MVP depth related analysis.

\begin{tabular}{|lllll|}
\hline AFV & $\begin{array}{l}\text { MVP } \\
\text { Depth } \\
(\mathrm{cm})\end{array}$ & $\begin{array}{l}\text { Present } \\
\text { study } \\
\%\end{array}$ & $\begin{array}{l}\text { Chamberlin } \\
\text { et al } \\
\%\end{array}$ \\
\hline $\begin{array}{l}\text { Polyhydra- } \\
\text { mnios }\end{array}$ & $>8 \mathrm{~cm}$ & 02 & 01.32 & 03 \\
\hline Normal & $2.1-8 \mathrm{~cm}$ & 130 & 86.10 & 94 \\
\hline Marginal & $1-2 \mathrm{~cm}$ & 19 & 12.58 & 02 \\
\hline Decreased & $<1 \mathrm{~cm}$ & & & 01 \\
\hline
\end{tabular}

Table 4 shows that when we apply 2-D POCKET for AFV assessment 103 patients were found to have oligoamnios, 43 patients were found to have normal amount and 05 patients were found to have polyhydramnios.
Table 4: AVF by 2 Diameter POCKET $(\mathrm{cm})$ related analysis.

\begin{tabular}{|lll|}
\hline 2-Diameter POCKET & $\begin{array}{l}\text { Present study } \\
(\mathbf{N = 1 5 1})\end{array}$ & $\%$ \\
\hline $0-15 \mathrm{~cm}^{2}$ & $(103)$ & 68.22 \\
\hline $15.1-50 \mathrm{~cm}^{2}$ & $(43)$ & 28.48 \\
\hline$>50 \mathrm{~cm}^{2}$ & $(05)$ & 03.31 \\
\hline
\end{tabular}

Table 5 shows comparision of different methods of amniotic fluid volume assessment.

Results of subjective and abdominal palpation methods are in agreement with that of the AFI whereas results of MVP DEPTH and 2D POCKET methods were different from AFI.

Table 5: Critical analysis of different methods in present study.

\begin{tabular}{|c|c|c|c|c|c|c|c|c|c|}
\hline \multirow{3}{*}{ Aligoamnios } & \multicolumn{9}{|l|}{ Methods } \\
\hline & $\begin{array}{l}\text { AFI } \\
\mathrm{N}=151(\%)\end{array}$ & \multicolumn{2}{|c|}{$\begin{array}{l}\text { MVP-depth } \\
\text { N=151 (\%) }\end{array}$} & \multicolumn{2}{|c|}{$\begin{array}{l}\text { 2-D-pocket } \\
\mathrm{N}=151(\%)\end{array}$} & \multicolumn{2}{|c|}{$\begin{array}{l}\text { Subjective } \\
\mathrm{N}=151(\%)\end{array}$} & \multicolumn{2}{|c|}{$\begin{array}{l}\text { Abdominal palpation } \\
\mathrm{N}=151(\%)\end{array}$} \\
\hline & $17 \quad(11.26)$ & - & - & 103 & $(68.22)$ & 15 & $(09.93)$ & 11 & $(07.28)$ \\
\hline Border line & (18.54) & 19 & $(12.58)$ & - & - & 24 & $(15.89)$ & 22 & (14.57) \\
\hline Adequate & $(62.92)$ & 130 & $(86.10)$ & 43 & $(28.48)$ & 103 & $(68.22)$ & 103 & $(68.22)$ \\
\hline Polyhydramnios & $(07.28)$ & 02 & $(01.32)$ & 05 & $(03.31)$ & 09 & $(05.96)$ & 15 & (09.93) \\
\hline
\end{tabular}

Table 6: Different methods as a screening test in patients with normal AFV by AFI.

\begin{tabular}{|c|c|c|c|c|c|c|}
\hline \multirow{3}{*}{ Screening Test } & \multirow{2}{*}{\multicolumn{2}{|c|}{$\begin{array}{l}\text { Sensitivity } \\
N=103\end{array}$}} & \multirow{2}{*}{\multicolumn{2}{|c|}{$\begin{array}{l}\text { Specificity } \\
\mathbf{N}=47\end{array}$}} & \multicolumn{2}{|c|}{ Predictive value } \\
\hline & & & & & \multirow{2}{*}{ Positive \% } & \multirow{2}{*}{ Negative $\%$} \\
\hline & No & $\%$ & No & $\%$ & & \\
\hline MVPD ( $2 \mathrm{~cm}$ rule) & 95 & 100 & 21 & 37.50 & 73.08 & 100 \\
\hline 2-diameter pocket & 35 & 36.84 & 48 & 85.71 & 81.40 & 44.44 \\
\hline Subjective & 83 & 87.37 & 36 & 64.29 & 80.58 & 75.00 \\
\hline abdominal-palpation & 72 & 75.79 & 25 & 44.64 & 69.90 & 52.08 \\
\hline
\end{tabular}




\section{DISCUSSION}

Before 1975, discussions of amniotic fluid volume assessment in the obstetric literature were limited to observations of the quantity of fluid released after rupture of membranes. With the advent of ultrasound, AFV assessment has gained reasonable accuracy and establishment of standards. In the course of pregnancy, the subject of amniotic fluid is seldom considered unless there is some abnormality e.g. polyhydramnios, oligohydramnios or the occurrence of meconium staining. However, when amniotic fluid volume is abnormal, perinatal morbidity and mortality are greatly increased. Critical analysis of each individual method of amniotic fluid volume for its sensitivity and specificity with regard to diagnosis of oligohydramnios, polyhydramnios and euamnios is done.

Table 7: Different methods as a screening test in patients with polyhydramnios by AFI.

\begin{tabular}{|c|c|c|c|c|c|c|}
\hline \multirow{2}{*}{ Screening Test } & \multicolumn{2}{|c|}{ Sensitivity $N=103$} & \multicolumn{2}{|c|}{ Specificity $N=47$} & \multicolumn{2}{|c|}{ Predictive value } \\
\hline & No & . & No & $\%$ & Positive \% & Negative $\%$ \\
\hline MVPD ( $2 \mathrm{~cm}$ rule) & 2 & 18.18 & 140 & 100 & 100 & 93.96 \\
\hline 2-diameter pocket & 4 & 36.36 & 139 & 99.29 & 80 & 95.21 \\
\hline Subjective & 6 & 54.55 & 137 & 97.86 & 66.67 & 96.48 \\
\hline abdominal-palpation & 6 & 54.55 & 131 & 93.57 & 40 & 96.32 \\
\hline
\end{tabular}

Table 8: Different methods as a screening test in patients with oligoamnios by AFI.

\begin{tabular}{|c|c|c|c|c|c|c|}
\hline \multirow{2}{*}{ Screening Test } & \multicolumn{2}{|c|}{ Sensitivity $\mathbf{N}=17$} & \multicolumn{2}{|c|}{ Sensitivity N=134 } & \multicolumn{2}{|c|}{ Predictive value } \\
\hline & No & $\%$ & No & 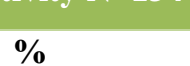 & Positive \% & Negative $\%$ \\
\hline 1-cm X 1-cm pocket & 02 & 11.76 & 134 & 100 & 100 & 89.93 \\
\hline 2-cm X 2-cm pocket & 16 & 94.12 & 122 & 91.01 & 57.14 & 99.19 \\
\hline MVPD (2 cm rule) & 15 & 88.24 & 130 & 97.01 & 78.95 & 98.48 \\
\hline 2-diameter pocket & 17 & 100.00 & 48 & 35.82 & 16.51 & 64.18 \\
\hline Subjective & 11 & 64.71 & 130 & 97.01 & 73.33 & 95.59 \\
\hline Abdominal-palpation & 08 & 47.06 & 127 & 97.78 & 53.33 & 93.38 \\
\hline
\end{tabular}

Table 1 shows that present study is in agreement with that of the Phalen et al and the difference is statistically insignificant. ${ }^{8}$ In present study number of patients having less liquor is $45(29.80 \%)$. This is due to the high incidence of causes responsible for that in our community e.g. IUGR, maternal disorders like anemia, pregnancy induced hypertension, post maturity, hypovolemia, ingestion of medications.

Table 2 shows that abnormalities in AFV are highest in the age group of 20-24 and next in the age group of 2529. This is because percentage of patients from these age groups $(20-29)$ is very high $(80.13 \%)$. This shows that Indian women usually complete their family before the age of 30 years as well as decreasing trend of teenage pregnancy. Table 3 shows that present study is in agreement with that of the Chamberlain et al and the difference is statistically insignificant. ${ }^{1,3}$ Number of patient with oligoamnios is zero which is due to poor sensitivity of this method of assessment as compare to AFI. Table 4 shows that when we apply 2-D POCKET for AFV assessment, a very large number of patients (103) were found to have oligoamnios. This suggests lower specificity of this method. Table 5 shows that experienced sinologist can predict AFV by subjective assessment with reasonable accuracy which can be seen from the results of this method and that of the AFI.

Results of subjective analysis and abdominal palpation methods are in agreement with that of the AFI. This shows that these methods have good predictive values, whereas MVP depth and 2-diameter pocket methods have poor predictive values. Subjective and MVP depth methods are more predictive of oligoamnios. Table 6 shows that MVP depth has excellent sensitivity (100\%) but poor specificity (37.50\%). It is good for screening but poor for diagnosis as large number of patients are missed. 2-diameter pocket has excellent specificity (85.71) but poor sensitivity (36.84), so it is good for diagnosis but poor for screening.

Subjective method has excellent sensitivity (87.37) but poor specificity (64.29\%), so it can be used for screening but for diagnosis it can be used in cinjunction with other methods. Abdominal palpation has poor sensitivity as well as sensitivity. Table 7 shows that MVP depth and 2diameter pocket have good specificity but low sensitivity, so good for diagnosis. Subjective and abdominal 
palpation methods have equally good specificity and sensitivity, so good for screening. Table 08 shows that $2 \mathrm{~cm} \times 2 \mathrm{~cm}$ method has excellent sensitivity (94.12\%) as well as specificity (91.01), so it can be used as a single test for screening as well as diagnosis.

MVP depth has excellent specificity $(97.01 \%)$ as well as sensitivity $(88.24 \%)$ so it can be used as an alternative test for diagnosis as well as for screening. 2-D pocket has excellent sensitivity (100\%) but very poor specificity $(35.82 \%)$ so it can be used for screening but not for diagnosis as predictive value of a positive test in patients with oligoamnios is only $16.51 \%$. Subjective method has excellent specificity $(97.01 \%)$ but poor sensitivity (64.71\%), which makes it better for diagnosis but for screening it can be used in conjuction with other methods.

Abdominal palpation has excellent specificity (94.78\%) but poor sensitivity $(47.06 \%)$ which makes it good for diagnosis but not for screening. Despite the reproducibility, Moore et al showed that well trained observers would subjectively identify patients with oligohydramnios with an intraclass correlation efficient of $0.81 .{ }^{9}$ The study of Halperin et al in which experienced sonographers were assigned patients to groups with normal, borderline low or reduced amniotic fluid volume, found that more experienced sonographers had significantly higher intraobserver correlation scores $(K=$ 0.94 vs $\mathrm{K}=.63) .{ }^{10}$

\section{CONCLUSION}

The optimal technique for amniotic fluid volume assessment should reproducibly assess AFV and should correlate well with abnormal fetal and maternal physiologic state. It should also be simple enough to be learned and used readily clinically. Present study draws following conclusions.

Almost all methods are reasonably effective in assessment of normal AFV. For assessment of oligoamnios, $2 \mathrm{~cm} \mathrm{X} 2 \mathrm{~cm}$ pocket and MVP depth of $2 \mathrm{~cm}$ pocket rule methods are most suitable whereas other methods were found to be inappropriate. For assessment of polyhydramnios, with regard to specificity MVP depth ( $8 \mathrm{~cm}$ rule) is reliable but with regard to sensitivity subjective assessment and abdominal palpation are more reliable. When we compare all methods of AFV assessment, AFI and MVP depth $(2 \mathrm{~cm}$ rule) have better correlation. Hence these methods are suggested in current clinical setting. However, randomized controlled trials involving large sample size is needed to draw further conclusions.
Funding: No funding sources

Conflict of interest: None declared

Ethical approval: The study was approved by the Institutional Ethics Committee

\section{REFERENCES}

1. Chamberlain MB, Manning FA, Morrison I. Ultrasound evaluation of amniotic fluid I. The evaluation of marginal and decreased amniotic fluid volume to perinatal outcome. Am J Obstet Gynecol. 1984;150:245-9.

2. Doubilet PM, Benson CB. Ultrasound evaluation of amniotic fluid. Ultrasonography in obstetrics and gynecology. Philadelphia; 1994:475-486.

3. Chamberlain MB, Manning FA, Morrison I. Ultrasound evaluation of amniotic fluid volume II. The relationship of increased amniotic fluid volume to perinatal outcome. Am J Obstet Gynecol. 1984;150:250-4.

4. Dildy GA, Lira N, Moise KJ, Riddle GD, Deter RL. Amniotic fluid volume assessment: comparison of ultrasonographic estimates versus direct measurements with a dye-dilution technique in human pregnancy. Am J Obstet Gynecol. 1992;167(4):986-94.

5. Crowley P. Non-quantitative estimation of amniotic fluid volume in suspected prolonged pregnancy. J Perinat Med. 1980;8:249-51.

6. Tempkin BG, Bizjak PM. Second and third trimester obstetrics Sonography, R.A. Curry, $2^{\text {nd }}$ ed. Elseveir Science. St. Louis;2004:320-355

7. Manning FA, Platt LD, Sipos L. Antepartum fetal evaluation: development of a fetal biophysical profile. Am J Obstet Gynecol. 1980;136(6):787-95.

8. Phelan JP, Ahn MO, Smith CV, Rutherford SE, Anderson E. Amniotic fluid index measurements during pregnancy. J Reproduc Med. 1987;32(8):6014.

9. Moore TR, Longo J, Leopold GR, Casola G, Gosink BB. The reliability and predictive value of an amniotic fluid scoring system in severe secondtrimester oligohydramnios. Obstet Gynecol. 1989;73(5):739-42.

10. Halperin ME, Fong KW, Zalev AH, Goldsmith $\mathrm{CH}$. Reliability of amniotic fluid volume estimation from ultrasonograms: intra observer and interobserver variation before and after the establishment of criteria. Am J Obstet Gynecol. 1985;153(3):264-7.

Cite this article as: Gandhi MR. A prospective study of critical methods of amniotic fluid volume assessment. Int J Reprod Contracept Obstet Gynecol 2017;6:3021-5. 